\title{
Anisotropy of ongoing neural activity in the primate visual cortex
}

This article was published in the following Dove Press journal:

Eye and Brain

23 September 2014

Number of times this article has been viewed

\section{Alexander Maier ${ }^{1}$ \\ Michele A Cox' \\ Kacie Dougherty' \\ Brandon Moore' \\ David A Leopold ${ }^{2}$}

'Department of Psychology,

College of Arts and Science, Vanderbilt University, Nashville, TN, USA; ${ }^{2}$ Section on Cognitive Neurophysiology and Imaging,

National Institute of Mental Health,

National Institute of Health, Bethesda, MD, USA
Correspondence: Alexander Maier Department of Psychology, College of Arts and Science, Vanderbilt University, III 2 I st Ave S., WH 008, Nashville, TN 37240, USA

Email alex.maier@vanderbilt.edu

\begin{abstract}
The mammalian neocortex features distinct anatomical variation in its tangential and radial extents. This review consolidates previously published findings from our group in order to compare and contrast the spatial profile of neural activity coherence across these distinct cortical dimensions. We focus on studies of ongoing local field potential (LFP) data obtained simultaneously from multiple sites in the primary visual cortex in two types of experiments in which electrode contacts were spaced either along the cortical surface or at different laminar positions. These studies demonstrate that across both dimensions the coherence of ongoing LFP fluctuations diminishes as a function of interelectrode distance, although the nature and spatial scale of this falloff is very different. Along the cortical surface, the overall LFP coherence declines gradually and continuously away from a given position. In contrast, across the cortical layers, LFP coherence is discontinuous and compartmentalized as a function of depth. Specifically, regions of high LFP coherence fall into discrete superficial and deep laminar zones, with an abrupt discontinuity between the granular and infragranular layers. This spatial pattern of ongoing LFP coherence is similar when animals are at rest and when they are engaged in a behavioral task. These results point to the existence of partially segregated laminar zones of cortical processing that extend tangentially within the laminar compartments and are thus oriented orthogonal to the cortical columns. We interpret these electrophysiological observations in light of the known anatomical organization of the cortical microcircuit.
\end{abstract}

Keywords: visual cortex, connectivity, local field potential, coherence

\section{Introduction}

The adoption of the Latin "cortex" to describe the outer gray matter covering of the brain paints this structure as a thin bark or rind enveloping the remainder of the brain. ${ }^{1}$ The cerebral cortex, however, is not a homogeneous, two-dimensional mantle. Rather, the cortex is a multilayered sheet with rich laminar interconnections. ${ }^{2-6}$ Accordingly, there is a notable spatial anisotropy in the radial (laminar) arrangement of cortical cell bodies and their projections. What is the consequence of this anatomical organization for neural activity patterns measured in the cortical microcircuit? For example, might the intrinsically generated, spontaneous activity be highly coordinated within a cortical column but relatively independent between cortical columns? Addressing this type of question requires simultaneous measurements at known spatial intervals in the cortex. Recent advances in neurophysiological techniques have exploited high-density multicontact recordings of extracellular electrical activity in vivo ${ }^{7-9}$ to facilitate comparison of neuronal signals collected simultaneously. This approach allows one to assess the spatial characteristics of 
neural coordination between known positions within the three-dimensional cortical extent.

In this review, we highlight key findings from our group that shed light on neuronal activity patterns within the three-dimensional volume of the cerebral cortex. We focus on primate visual cortex as a model sensory system with well described anatomy that has been the subject of a large body of neurophysiological studies. To contextualize current work, we first provide an overview of the cortex's anatomical features as well as methods to assess spatial coordination of neural processes. We then briefly summarize neurophysiological data that demonstrate the fundamental anisotropy of neural coherence in the tangential versus radial dimensions of the cortex.

\section{Distinct anatomical features in the radial and tangential dimensions}

As early as the 19th century, neuroanatomists began to parcel the mammalian cortical sheet across the tangential dimension into distinct areas that differ in their cytoarchitecture..$^{10}$ These areas were subsequently shown to exhibit distinct patterns of connectivity and neuronal function. Some of the neocortical areas defined in these ways are conserved among primates and to some extent among other mammalian orders in their topological position and pattern of interareal projections. ${ }^{11-13}$ Within each cortical area, certain axonal projections do not enter the white matter, but instead remain in the neuropil. These "horizontal" connections, which are laterally extending branches of pyramidal cell axons, ${ }^{14,15}$ are marked by a high degree of functional specificity and often target neurons or columns with broadly similar response properties. ${ }^{16}$ The extent of tangential cortical distance covered by these horizontal connections varies considerably between areas and increases at higher stages in the cortical hierarchy. ${ }^{17}$ Whereas axons in the primary visual cortex (V1) may reach $2 \mathrm{~mm},{ }^{18}$ those in the inferior temporal cortical area (TE) have been shown to project up to $9 \mathrm{~mm} .{ }^{19}$ Horizontal connections tend to be reciprocal, ${ }^{20}$ commonly remain within their cortical layer, ${ }^{21,22}$ and are largely excitatory both in origin as well as in their postsynaptic targets. ${ }^{23,24}$ Functionally speaking, horizontal connections are mostly modulatory in nature, ${ }^{25}$ and likely contribute to the high activity coherence measured over short distances in the cortex (see Measuring the reach of activity coherence in the cortex).

Early neuroanatomists also recognized the importance of the radial dimension to the composition of the cortex, with sheets of cells arranged in segregated layers that form early in development. ${ }^{26,27}$ Four to six cytoarchitectonic layers can be visualized with a variety of histological staining techniques (Figure 1A). The exact delineation of the respective layer boundaries depends somewhat on the type of histological stain used, the cortical areas examined, and the animal species. ${ }^{28}$ The resulting variability in layer count has prompted the adoption of slightly different labeling schemes over the years, ${ }^{29,30}$ but broad consensus follows neuroanatomist Korbinian Brodmann's original plan of dividing the neocortex into six major laminae..$^{10}$ These individual layers are further grouped into three visibly distinct laminar domains. Layer 4 and its various sublayers are commonly denoted as granular, since the high density of spiny cell bodies in this domain appears grainy in Nissl-type histological stains. Superficial layers 1-3 thus occupy a supragranular position, whereas deeper layers 5 and 6 are infragranular. ${ }^{31}$

In contrast with the pronounced morphological differences between cortical layers, functional responses across layers are similar, and have given rise to the concept of a cortical column. ${ }^{32-36}$ The cortical column is conceptualized as a repeated motif of radial units, each supported by a stereotypical pattern of intrinsic connections, ${ }^{37}$ the functional homogeneity of which is often considered a universal and critical feature of the cerebral cortex..$^{38}$ In the primary visual cortex, for example, the same basic orientation and eye preferences can be observed across layers of a given column. ${ }^{39}$ Moreover, studies point to a basic model of sequential excitation across particular laminae within a column following

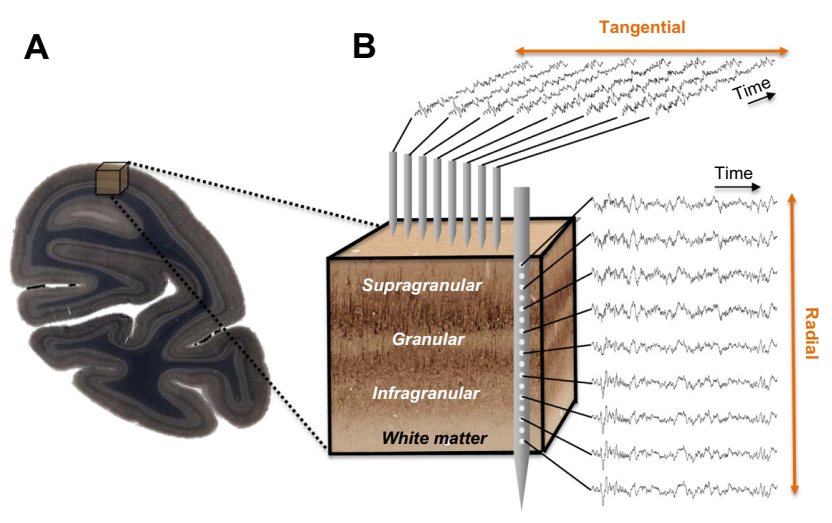

Figure I Neurophysiological measures within a cortical volume.

Notes: (A) In most neurophysiological preparations, microelectrodes are inserted into the gray matter and time-varying voltage is measured against a nearby reference. (B) If recordings are performed at more than one location simultaneously, one can compare signals along the radial dimension across the cortical layers or along the tangential dimension that runs in parallel with the cortical surface. While radial recordings are often performed with linear multielectrode arrays that consist of multiple electrode contacts that run along a single electrode shaft, tangential recordings typically require two or more needle-shaped microelectrodes spaced apart. 
the arrival of sensory information from the thalamus. ${ }^{40,41}$ This model suggests that thalamic input to cortical layer 4 is relayed to the supragranular layers, where it is integrated with various types of modulatory signals, ${ }^{2,42-44}$ and then relayed to the infragranular layers. Infragranular neurons send local projections to granular and supragranular targets in the same cortical column, as well as long-range projections to cortical and subcortical structures inside and outside of the telencephalon. ${ }^{45}$ While there are many ways in which information can be integrated across columns, and the generality of functional columns in the cortex remains a topic of debate, ${ }^{46-58}$ this idealized microcircuit model emphasizing intracolumnar processing has played an important role in understanding the complexity of cortical projection patterns and their bearing on measured sensory signals. ${ }^{59,60}$

To summarize, the anatomical organization of the cortex varies considerably across both the radial and tangential dimensions. Within a given area, horizontal connections account for much of the tangential connectivity, whereas the interlaminar projections account for the radial connectivity.

\section{Measuring the reach of activity coherence in the cortex}

The local field potential (LFP) represents a collective measure of neural activity, including subthreshold synaptic processes that are synchronized in space and time.$^{61-66}$ Its inherent heterogeneity limits the precision of the LFP for probing details of microcircuit function. ${ }^{67,68}$ Nonetheless, empirical studies have shown that both sensory-evoked LFP responses ${ }^{69}$ and high frequency "gamma" range $(>30 \mathrm{~Hz})$ LFP power ${ }^{70,71}$ are often well correlated with local spiking activity in the cortex. Thus, it is possible to use the LFP, and in particular its upper frequency range, to study neural coordination at different spatial scales. However, a conspicuous feature of the LFP signal is its $1 / f^{\beta}$ spectral distribution, where $f$ denotes frequency and $\beta$ is the exponent of a power law. ${ }^{72,73}$ This means that the high frequency gamma range is at least one order of magnitude smaller in amplitude than the lower frequency fluctuations, which dominate the raw signal fluctuations.

Experimental approaches to measure the spatiotemporal coordination of neural signals involve simultaneous electrophysiological measurement from multiple electrodes (Figure 1B). Having obtained such simultaneous signals, most attempts to evaluate the interaction between sites amount to a measure of temporal correlation of two or more neural signals. To this end, neurophysiologists employ a wide range of correlational measures that will not be reviewed here.
Here we describe one common measure, the mean squared coherence, which measures signal correlation as a function of frequency. Whether or not frequency is a natural domain across which to divide neural signals is a matter of debate, although this practice is very common. What is clear is that doing so allows one to isolate aspects of the LFP signal that correlate with particular behavioral or neural events, such as the close relationship between the local spiking and the low amplitude gamma range mentioned above. It is important to mention that the precision of LFP coherence as a measure of neural coordination is limited by electric volume conduction. In contrast with spiking activity, where the signal source can be identified as one or more nearby neurons, the LFP reflects an ambiguous combination of superimposed local and distant neural events. ${ }^{65,67}$

In the following section, we use mean squared coherence as a measure to assess the coherence across the different cortical dimensions. Of particular interest is how the pronounced anatomical anisotropy of the cortex described in the previous section influences the spatial coordination of neural activity across these different dimensions.

\section{Distinct laminar sheets of coherent LFP}

Given the columnar cortical architecture outlined above, one might predict that LFP coherence would also follow a columnar pattern. Would intrinsic LFP fluctuations show more coherence within a column than between columns? Interestingly, the data suggest nearly the opposite: the spatial stretch of LFP coordination is perpendicular to the cortical columns. The LFP is spatially coherent along zones extending tangentially, but is sharply discontinuous within a column.

Between electrodes spaced tangentially along the cortical surface, LFP coherence falls off continuously and in a frequency-dependent fashion. One study of the macaque visual cortex examined the coherence of ongoing activity across distances spanning from a few hundred microns to over a centimeter as monkeys rested idly in a dark room. ${ }^{74}$ The main findings of that study were that LFP coherence falls off monotonically, and that the falloff is steeper for high frequency than for low frequency LFP components (Figures $2 \mathrm{~A}$ and $3 \mathrm{~A}$ ). A subsequent study showed that this falloff reflects the cortical distance rather than the absolute distance between the electrode contacts, since LFP coherence between electrodes placed on either side of a sulcus showed a drop reflecting the span of the infolded cortex. ${ }^{75}$ This general pattern of coherence is qualitatively similar during rest and when monkeys perform a simple behavioral task (ie, when they are 
A
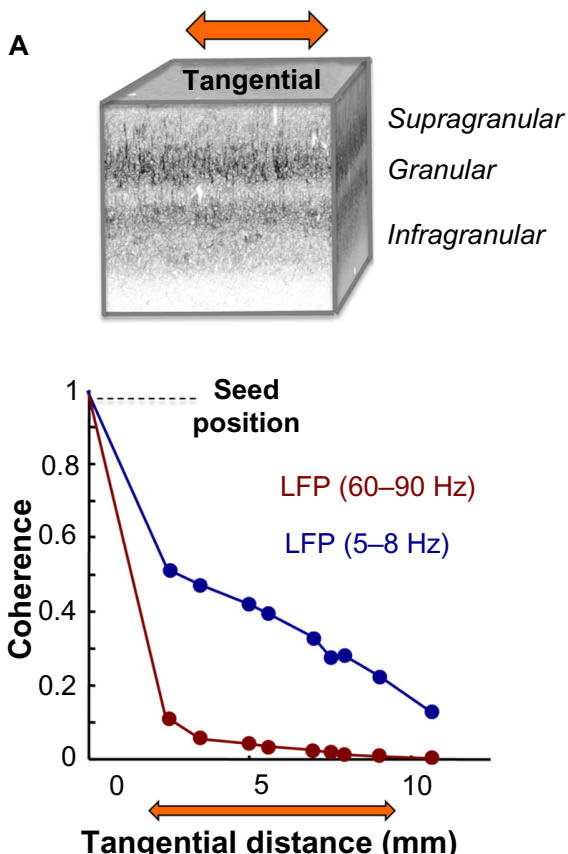

B
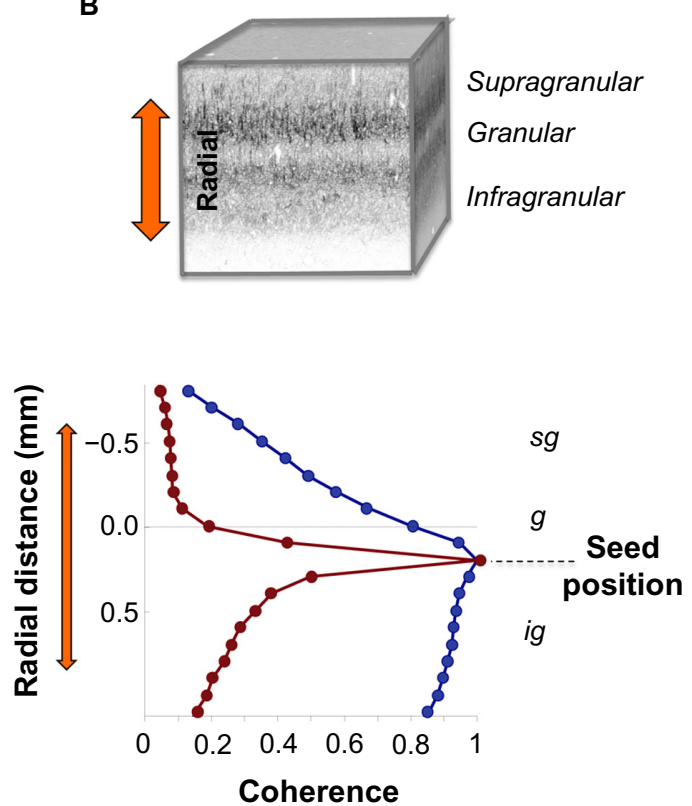

Figure 2 LFP coherence as a function of tangential and radial distance.

Notes: (A) LFP coherence (ordinate) is computed between a seed electrode in the primary visual cortex and several neighboring electrodes that are spaced at varying intervals of tangential cortical distance (abscissa). Each point of the graph depicts the LFP coherence value measured at that cortical recording location with respect to the seed position. High frequency LFP coherence (red line) and low frequency LFP coherence (blue line) are shown separately. Note the difference in tangential falloff of LFP coherence, indicating that slow neural processes are more widespread along the cortical mantle compared with locally confined fast neural activity (see Leopold et al ${ }^{74}$ for details and statistics). (B) LFP coherence as a function of radial (laminar) cortical distance. Each point of the graph corresponds to an electrode contact position of a linear electrode array that was placed to record neural activity across all of primary visual cortex's layers between the pia mater and the white mater. LFP coherence for each of these recording locations is computed against the electrode contact in the infragranular layers marked as the seed position. Dashed horizontal line marks the transition zone between the granular and infragranular layers. Note that for the same radial cortical distance LFP coherence remains higher in the infragranular compartment than in the supragranular compartment (see Maier et $\mathrm{al}^{79}$ for details and statistics).

Abbreviations: LFP, local field potential; sg, supragranular; g, granular; ig, infragranular.

behaviorally engaged) and is comparable in amplitude and slope between areas V1, V2, and V4. The results are consistent with other findings that show steep $(<5 \mathrm{~mm})$ falloff in high frequency LFP and spiking ${ }^{76}$ and more gradual $(>5 \mathrm{~mm})$ falloff in low frequency LFP coherence as a function of lateral cortical distance. ${ }^{67,69,74,77}$

In the radial dimension, coherence has recently been assessed between contacts occupying different laminar
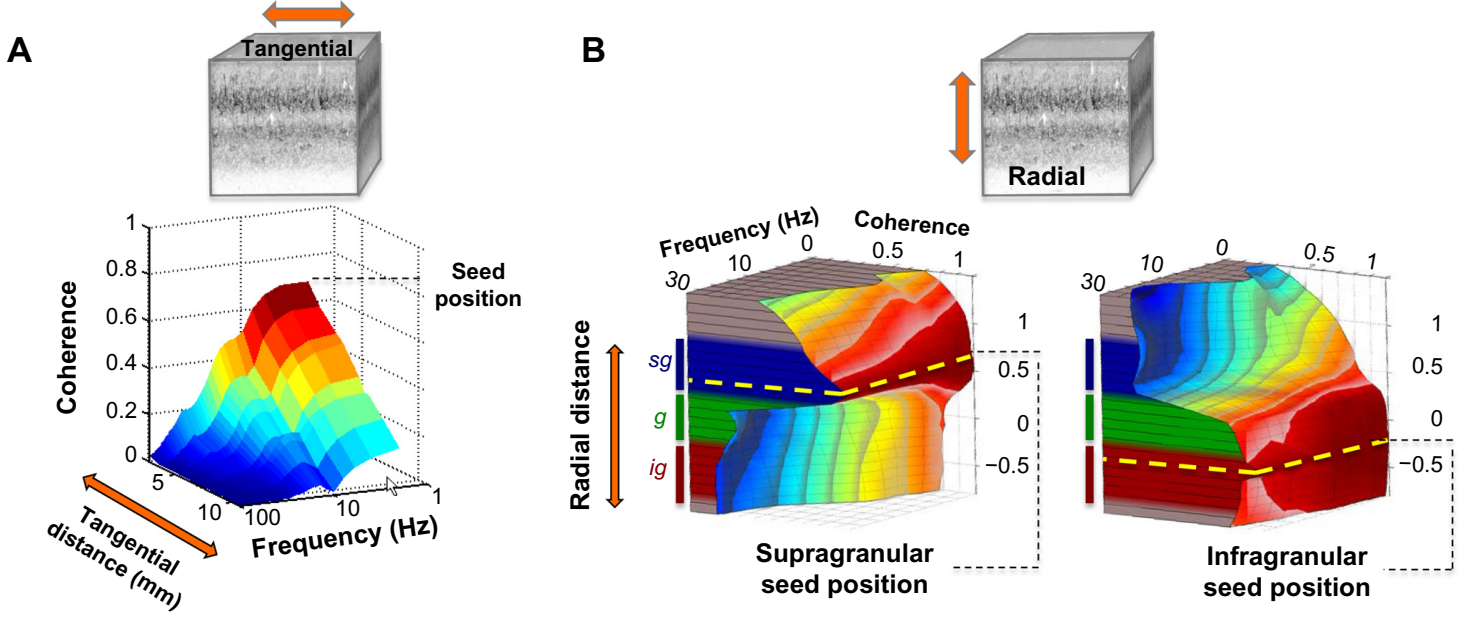

Figure 3 Volumetric profile of LFP coherence.

Notes: (A) LFP coherence is shown as a function of frequency and tangential distance relative to a seed electrode at distance 0 . Note the monotonic falloff of LFP coherence with tangential cortical distance (see Leopold et $\mathrm{al}^{74}$ for details). (B) LFP coherence is shown as a function of frequency and cortical depth. A seed electrode (yellow dashed line) was chosen in the supragranular layers (left) or in the infragranular layers (right), and coherence was computed as a function of frequency in spatial increments of 100 microns. Note the falloff of coherence for high frequency activity in the laminar compartment where the seed is located (see Maier et al ${ }^{79}$ for details).

Abbreviations: LFP, local field potential; sg, supragranular; g, granular; ig, infragranular. 
positions of the visual cortex. In contrast with the gradual falloff in LFP coherence along the cortical surface, the pattern of laminar coherence is discontinuous and strongly compartmentalized, particularly for frequency ranges below the gamma range (Figures 2B and 3B). ${ }^{78,79}$ Probing the spatial pattern of coherence from different seeds reveals two prominent laminar zones of coherent LFP activity separated by the deep granular layer, with one zone dominating within the superficial layers (1-3) and another zone in the deep layers (5 and 6). While the LFP coherence within these zones was high, that between them was nearly zero across a wide frequency range (see Figure 3B). This spatial structure was present in the ongoing activity both during rest and when the animals were engaged in a behavioral task. Taken together, the studies outlined above suggest that the spatial extent of ongoing LFP coherence spreads broadly in the tangential direction but is restricted in the radial direction. The resulting spatial pattern can be conceptualized as two laminar sheets of coherent activity (Figure 4), which constitutes a marked deviation from the radial anisotropy predicted by the columnar architecture described above.

The findings reviewed here point to an intricate threedimensional structure of neural coherence that reflects a laminar compartmentalization emerging when the brain is not explicitly stimulated (with the animals being either at rest or behaviorally engaged). Neither known interlaminar connections nor innervation from the thalamus or other cortical areas can fully explain the strictly separate domains of intracortical coherence $\mathrm{e}^{37,80-83} \mathrm{we}$ observed in the upper and lower cortical layers. Nonetheless, it is interesting to speculate that in the absence of specific sensory input (ie, during periods of rest or between sensory events), cortical activity may switch from a "columnar mode", where the sensory world is parsed by a large number of columnar units dedicated to extracting features of the world, to a "laminar mode", where functional differences in the tangential direction become less important, and activity segregates by layer.

What might be the basis for this laminar segregation of intrinsic activity? One possibility is that it reflects a division between intracortical versus thalamocortical interactions, with one contributing most strongly to the upper layer fluctuations and the other to the lower layer fluctuations. While clearly a speculation at this point, this explanation would account for the apparently separate laminar sheets of ongoing cortical activity that are largely uncoordinated across their boundary in the granular layer.

At first sight, our observation of radially anisotropic ongoing activity seems at odds with the columnar structure of stimulusevoked responses because of the apparent compartmentalization that has been reported in certain studies. However, several other neurophysiological phenomena have been described that exhibit similar laminar specificity. For example, neurophysiological recordings with multiple laminar electrodes inserted simultaneously into rat auditory cortex found that the spatial pattern of spiking activity within the supragranular layers was highly localized, sparse, and strongly specific to a small subset of
A

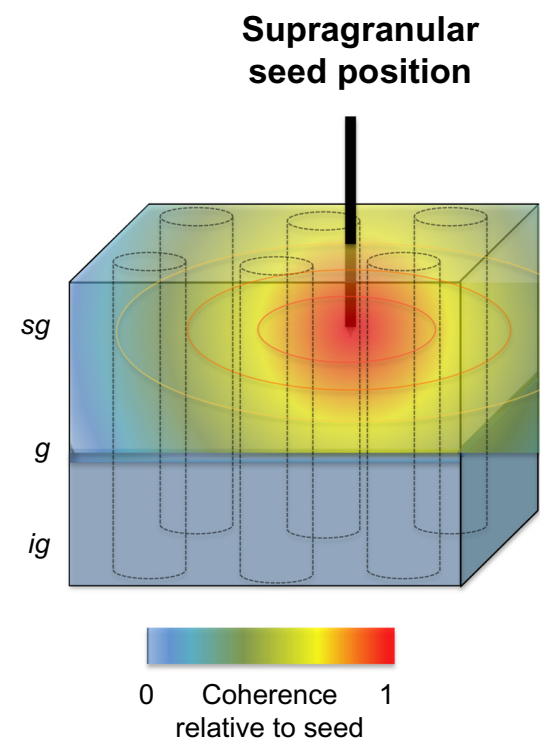

B

$$
\begin{aligned}
& \text { Infragranular } \\
& \text { seed position }
\end{aligned}
$$

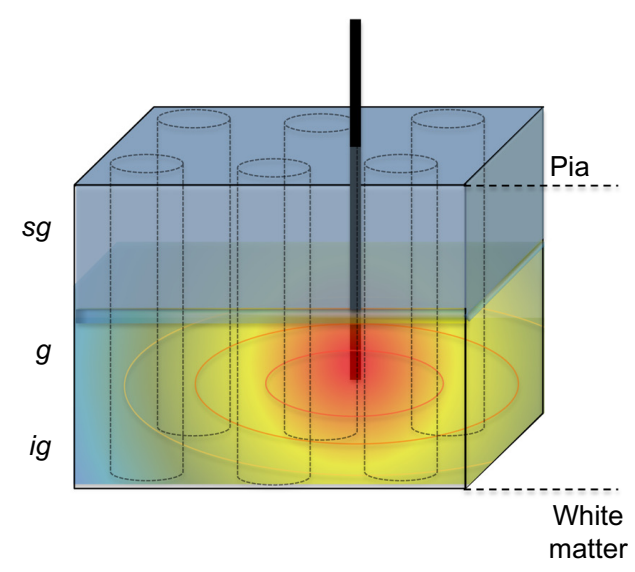

Figure 4 Schematic representation of the spatial anisotropy of LFP coherence along the tangential and radial dimensions of primate primary visual cortex.

Notes: (A) Graphic depicting the spatial falloff in LFP coherence with increasing distance from an electrode location in the supragranular layers. The three-dimensional expanse of the neocortical tissue sample is depicted as a blue-grayish box. Cortical columns are conceptualized as black cylinders. The electrode tip is indicated as "seed". (B) Same as A, but with electrode recording location in the deep (infragranular) layers of cortex.

Abbreviations: LFP, local field potential; sg, supragranular; g, granular; ig, infragranular. 
sensory stimuli. In contrast, spiking within the infragranular layers was widespread, constant, and promiscuous with respect to sensory stimulation. ${ }^{84}$ Furthermore, analysis of spontaneous LFP recorded in several visual areas found a peak of low frequency $(\sim 10 \mathrm{~Hz})$ coherence between LFP and multiunit spiking activity in the infragranular layers that was absent in the supragranular layers. ${ }^{85}$ In contrast, the recently described repeating patterns of clustered cortical synchronization termed "neuronal avalanches" seem to be confined to the superficial layers of primate sensory cortex. ${ }^{86}$ Stimulus-evoked LFP power ${ }^{87}$ and multiunit spiking activity $^{88}$ of macaque V1 have been demonstrated to exhibit pronounced anisotropies between upper and lower cortical layers. Moreover, reversible inactivation (cooling) of the supragranular layers of cat primary visual cortex had little influence, if any, on the response properties of infragranular neurons. ${ }^{89,90}$ Lastly, some authors have reported stark differences in orientation tuning between supragranular and infragranular neurons within the same cortical column of macaque visual cortex, ${ }^{91-93}$ which they interpreted as evidence for a functional dichotomy between deep and superficial layers. ${ }^{94}$

These results, taken together with the coherence differences in LFP discussed above, hint at a strict functional division between the main laminar compartments of visual cortex. The laminar pattern of LFP activity described here thus may provide a unique window on the spatiotemporal structure of neuronal population activity that warrants further investigation into the relationship between structure and function within the cortical microcircuitry.

\section{Acknowledgments}

Authors would like to thank V Casagrande for comments on an earlier version of the manuscript as well as G Adams for his contributions to the figures. This research was supported in part by the Intramural Research Program of the NIMH. MAC is supported by a NSFGRF grant (DGE-0909667). AM is supported by grants from the Whitehall and Alfred $\mathrm{P}$ Sloan Foundation.

\section{Disclosure}

The authors report no conflicts of interest in this work.

\section{References}

1. Monro A. The cortex of the encephalon. Anat Nerves. 1742;(14).

2. Gilbert CD. Microcircuitry of the visual cortex. Annu Rev Neurosci. 1983;6(1):217-247.

3. Shadlen MN, Newsome WT. Noise, neural codes and cortical organization. Curr Opin Neurobiol. 1994;4(4):569-579.

4. Reynolds JH. Mapping the microcircuitry of attention. Nat Neurosci. 2008;11(8):861-862.

5. Izhikevich EM, Edelman GM. Large-scale model of mammalian thalamocortical systems. Proc Natl Acad Sci U S A. 2008;105(9):3593-3598.
6. Bastos AM, Usrey WM, Adams RA, Mangun GR, Fries P, Friston KJ. Canonical microcircuits for predictive coding. Neuron. 2012;76(4): 695-711.

7. Lakatos P, Karmos G, Mehta AD, Ulbert I, Schroeder CE. Entrainment of neuronal oscillations as a mechanism of attentional selection. Science. 2008;320(5872):110-113.

8. Ulbert I, Halgren E, Heit G, Karmos G. Multiple microelectroderecording system for human intracortical applications. J Neurosci Methods. 2001;106(1):69-79.

9. Buzsáki G. Large-scale recording of neuronal ensembles. Nat Neurosci. 2004;7(5):446-451.

10. Brodmann K. Localisation in the Cerebral Cortex. New York, NY, USA: Springer; 2007.

11. Kaas JH. The evolution of brains from early mammals to humans. Wiley Interdiscip Rev Cogn Sci. 2013;4(1):33-45.

12. Kaas JH. Evolution of columns, modules, and domains in the neocortex of primates. Proc Natl Acad Sci U S A. 2012;109 Suppl 1: 10655-10660.

13. Alfano C, Studer M. Neocortical arealization: evolution, mechanisms, and open questions. Dev Neurobiol. 2013;73(6):411-447.

14. Gilbert CD, Wiesel TN. Clustered intrinsic connections in cat visual cortex. J Neurosci. 1983;3(5):1116-1133.

15. Rockland KS, Lund JS. Intrinsic laminar lattice connections in primate visual cortex. J Comp Neurol. 1983;216(3):303-318.

16. Stettler DD, Das A, Bennett J, Gilbert CD. Lateral connectivity and contextual interactions in macaque primary visual cortex. Neuron. 2002;36(4):739-750.

17. Amir Y, Harel M, Malach R. Cortical hierarchy reflected in the organization of intrinsic connections in macaque monkey visual cortex. J Comp Neurol. 1993;334(1):19-46.

18. Angelucci A, Levitt JB, Walton EJ, Hupe J-M, Bullier J, Lund JS. Circuits for local and global signal integration in primary visual cortex. $J$ Neurosci. 2002;22(19):8633-8646.

19. Tanigawa H, Wang Q, Fujita I. Organization of horizontal axons in the inferior temporal cortex and primary visual cortex of the macaque monkey. Cereb Cortex. 2005;15(12):1887-1899.

20. Angelucci A, Bullier J. Reaching beyond the classical receptive field of V1 neurons: horizontal or feedback axons? J Physiol Paris. 2003;97(2-3):141-154.

21. Malach R, AmirY, Harel M, Grinvald A. Relationship between intrinsic connections and functional architecture revealed by optical imaging and in vivo targeted biocytin injections in primate striate cortex. Proc Natl Acad Sci US A. 1993;90(22):10469-10473.

22. Yoshioka T, Blasdel GG, Levitt JB, Lund JS. Relation between patterns of intrinsic lateral connectivity, ocular dominance, and cytochrome oxidase-reactive regions in macaque monkey striate cortex. Cereb Cortex. 1996;6(2):297-310.

23. McGuire BA, Gilbert CD, Rivlin PK, Wiesel TN. Targets of horizontal connections in macaque primary visual cortex. J Comp Neurol. 1991;305(3):370-392.

24. Yoshimura Y, Sato H, Imamura K, Watanabe Y. Properties of horizontal and vertical inputs to pyramidal cells in the superficial layers of the cat visual cortex. J Neurosci. 2000;20(5):1931-1940.

25. Hirsch JA, Gilbert CD. Synaptic physiology of horizontal connections in the cat's visual cortex. J Neurosci. 1991;11(6):1800-1809.

26. Rakic P. Radial unit hypothesis of neocortical expansion. Novartis Found Symp. 2000;228:30-42.

27. Goetz M, Bolz J. Formation and preservation of cortical layers in slice cultures. J Neurobiol. 1992;23(7):783-802.

28. DeFelipe J, Alonso-Nanclares L, Arellano JI. Microstructure of the neocortex: comparative aspects. J Neurocytol. 2002;31(3-5):299-316.

29. Billings-Gagliardi S, Chan-Palay V, Palay SL. A review of lamination in area 17 of the visual cortex Macaca mulatta. J Neurocytol. 1974;3(5):619-629.

30. Marín-Padilla M. Cajal-Retzius cells and the development of the neocortex. Trends Neurosci. 1998;21(2):64-71. 
31. Zeki S, Shipp S. The functional logic of cortical connections. Nature. 1988;335(6188):311-317.

32. Mountcastle VB. The columnar organization of the neocortex. Brain. 1997;120(4):701-722.

33. Lübke J, Feldmeyer D. Excitatory signal flow and connectivity in a cortical column: focus on barrel cortex. Brain Struct Funct. 2007;212(1):3-17.

34. de Nó RL. Cerebral cortex: architecture, intracortical connections, motor projections. In: Fulton, JF, editor. Physiology of the Nervous System. 3rd ed. Oxford, UK: Oxford University Press; 1949.

35. Hubel DH, Wiesel TN. Uniformity of monkey striate cortex: a parallel relationship between field size, scatter, and magnification factor. J Comp Neurol. 1974;158(3):295-305.

36. Hubel DH, Wiesel TN. Ferrier lecture. Functional architecture of macaque monkey visual cortex. Proc $R$ Soc Lond B Biol Sci. 1977;198(1130):1-59.

37. Callaway EM. Local circuits in primary visual cortex of the macaque monkey. Аnпи Rev Neurosci. 1998;21(1):47-74.

38. Lund JS, Angelucci A, Bressloff PC. Anatomical substrates for functional columns in macaque monkey primary visual cortex. Cereb Cortex. 2003;13(1):15-24.

39. Hubel DH, Wiesel TN. Receptive fields and functional architecture of monkey striate cortex. J Physiol. 1968;195(1):215-243.

40. Bode-Greuel KM, Singer W, Aldenhoff JB. A current source density analysis of field potentials evoked in slices of visual cortex. Exp Brain Res. 1987;69(1):213-219.

41. Nowak LG, Munk MH, Girard P, Bullier J. Visual latencies in areas V1 and V2 of the macaque monkey. Vis Neurosci. 1995;12(2):371-384.

42. Rockland KS, Pandya DN. Laminar origins and terminations of cortical connections of the occipital lobe in the rhesus monkey. Brain Res. 1979;179(1):3-20.

43. Rockland KS, Virga A. Terminal arbors of individual "Feedback" axons projecting from area $\mathrm{V} 2$ to $\mathrm{V} 1$ in the macaque monkey: a study using immunohistochemistry of anterogradely transported Phaseolus vulgaris-leucoagglutinin. J Comp Neurol. 1989;285(1):54-72.

44. Anderson JC, Martin KA. The synaptic connections between cortical areas V1 and V2 in Macaque monkey. J Neurosci. 2009;29(36): $11283-11293$

45. Thomson AM, Bannister AP. Interlaminar connections in the neocortex Cereb Cortex. 2003;13(1):5-14.

46. Rockel AJ, Hiorns RW, Powell TP. The basic uniformity in structure of the neocortex. Brain. 1980;103(2):221-244.

47. Douglas RJ, Martin KA, Whitteridge D. A canonical microcircuit for neocortex. Neural Comput. 1989;1:480-488.

48. Lund JS, Yoshioka T, Levitt JB. Comparison of intrinsic connectivity in different areas of macaque monkey cerebral cortex. Cereb Cortex. 1993;3(2):148-162.

49. Rockland KS. Complex microstructures of sensory cortical connections. 1998;8(4):545-551.

50. Sawaguchi T, Kubota K. A hypothesis on the primate neocortex evolution: column-multiplication hypothesis. Int $J$ Neurosci. 1986;30(1-2):57-64.

51. Silberberg G, Gupta A, Markram H. Stereotypy in neocortical microcircuits. Trends Neurosci. 2002;25(5):227-230.

52. Herculano-Houzel S, Collins CE, Wong P, Kaas JH, Lent R. The basic nonuniformity of the cerebral cortex. Proc Natl Acad Sci U SA. 2008; 105(34):12593-12598.

53. Haug H. Brain sizes, surfaces, and neuronal sizes of the cortex cerebri: A stereological investigation of man and his variability and a comparison with some mammals (primates, whales, marsupials, insectivores, and one elephant). Am J Anat. 1987;180(2):126-142.

54. Rakic P. Confusing cortical columns. Proc Natl Acad Sci U S A. 2008;105(34):12099-12100.

55. Horton JC, Adams DL. The cortical column: a structure without a function. Philos Trans R Soc Lond B Biol Sci. 2005;360(1456): 837-862.

56. Nelson SB. Cortical microcircuits. Neuron. 2002;36(1):19-27.
57. Van Hooser SD. Similarity and diversity in visual cortex: is there a unifying theory of cortical computation? Neuroscientist. 2007;13(6):639-656.

58. Swindale NV. Is the cerebral cortex modular? Trends Neurosci. 1990;13(12):487-492.

59. Rockland KS, Drash GW. Collateralized divergent feedback connections that target multiple cortical areas. J Comp Neurol. 1996;373(4):529-548.

60. Felleman DJ, van Essen DC. Distributed hierarchical processing in the primate cerebral cortex. Cereb Cortex. 1991;1(1):1-47.

61. Rasch M, Logothetis NK, Kreiman G. From neurons to circuits: linear estimation of local field potentials. J Neurosci. 2009;29(44): 13785-13796.

62. Reimann MW, Anastassiou CA, Perin R, Hill SL, Markram H, Koch C. A biophysically detailed model of neocortical local field potentials predicts the critical role of active membrane currents. Neuron. 2013;79(2):375-390.

63. Einevoll GT, Kayser C, Logothetis NK, Panzeri S. Modelling and analysis of local field potentials for studying the function of cortical circuits. Nat Rev Neurosci. 2013;14(11):770-785.

64. Buzsáki G, Logothetis N, Singer W. Scaling brain size, keeping timing: evolutionary preservation of brain rhythms. Neuron. 2013;80(3):751-764.

65. Mitzdorf U. Current source-density method and application in cat cerebral cortex: investigation of evoked potentials and EEG phenomena. Physiol Rev. 1985;65(1):37-100.

66. Buzsáki G, Anastassiou CA, Koch C. The origin of extracellular fields and currents - EEG, ECoG, LFP and spikes. Nat Rev Neurosci. 2012;13(6):407-420.

67. Kajikawa Y, Schroeder CE. How local is the local field potential? Neuron. 2011;72(5):847-858.

68. Łęski S, Lindén H, Tetzlaff T, Pettersen KH, Einevoll GT. Frequency dependence of signal power and spatial reach of the local field potential. PLoS Comput Biol. 2013;9(7):e1003137.

69. Katzner S, Nauhaus I, Benucci A, Bonin V, Ringach DL, Carandini M. Local origin of field potentials in visual cortex. Neuron. 2009;61(1): 35-41.

70. Liu J, Newsome WT, Liu J. Local field potential in cortical area MT: stimulus tuning and behavioral correlations. J Neurosci. 2006;26(30):7779-7790.

71. Ray S, Maunsell JH. Different origins of gamma rhythm and highgamma activity in macaque visual cortex. $J$ Neurosci. 2011;9(4): e1000610.

72. Freeman WJ. Neurodynamics. London, UK: Springer; 2000.

73. Buzsáki G. Rhythms of the Brain. Oxford, UK: Oxford University Press; 2006.

74. Leopold DA, Murayama Y, Logothetis N. Very slow activity fluctuations in monkey visual cortex: implications for functional brain imaging. Cereb Cortex. 2003;13(4):422-433.

75. Leopold DA, Logothetis NK. Spatial patterns of spontaneous local field activity in the monkey visual cortex. Rev Neurosci. 2003;14(1-2): 195-205.

76. Smith MA, Kohn A. Spatial and temporal scales of neuronal correlation in primary visual cortex. $J$ Neurosci. 2008;28(48): 12591-12603.

77. Canolty RT. Spatiotemporal dynamics of word processing in the human brain. Front Neurosci. 2007;1(1):185-196.

78. Harris KD, Thiele A. Cortical state and attention. Nat Rev Neurosci. 2011;12(9):509-523.

79. Maier A, Adams GK, Aura C, Leopold DA. Distinct superficial and deep laminar domains of activity in the visual cortex during rest and stimulation. Front Syst Neurosci. 2010;4(31):1-11.

80. Lund JS. Anatomical organization of macaque monkey striate visual cortex. Annu Rev Neurosci. 1988;11(1):253-288.

81. Yamamori T, Rockland KS. Neocortical areas, layers, connections, and gene expression. Neurosci Res. 2006;55(1):11-27.

82. Marion R, Li K, Purushothaman G, Jiang Y, Casagrande VA. Morphological and neurochemical comparisons between pulvinar and V1 projections to V2. J Comp Neurol. 2013;521(4):813-832. 
83. Kätzel D, Zemelman BV, Buetfering C, Wölfel M, Miesenböck G. The columnar and laminar organization of inhibitory connections to neocortical excitatory cells. Nat Neurosci. 2011;14(1):100-107.

84. Sakata S, Harris KD. Laminar structure of spontaneous and sensoryevoked population activity in auditory cortex. Neuron. 2009;64(3): 404-418.

85. Bollimunta A, Chen Y, Schroeder CE, et al. Neuronal mechanisms of cortical alpha oscillations in awake-behaving macaques. J Neurosci. 2008;28(40):9976-9988.

86. Petermann T, Thiagarajan TC, Lebedev MA, Nicolelis MA, Chialvo DR, Plenz D. Spontaneous cortical activity in awake monkeys composed of neuronal avalanches. Proc Natl Acad Sci USA. 2009;106(37): 15921-15926.

87. Maier A, Aura CJ, Leopold DA. Infragranular sources of sustained local field potential responses in macaque primary visual cortex. J Neurosci. 2011;31(6):1971-1980.

88. Xing D, Yeh C-I, Burns S, et al. Laminar analysis of visually evoked activity in the primary visual cortex. Proc Natl Acad Sci U S A. 2012;109(34):13871-13876.
89. Weyand TG, Malpeli JG, Lee C, Schwark HD. Cat area 17. III. Response properties and orientation anisotropies of corticotectal cells. J Neurophysiol. 1986;56(4):1088-1101.

90. Schwark HD, Malpeli JG, Weyand TG, Lee C. Cat area 17. II. Response properties of infragranular layer neurons in the absence of supragranular layer activity. J Neurophysiol. 1986;56(4):1074-1087.

91. Bauer R, Dow DB, Snyder AZ, Vautin R. Orientation shift between upper and lower layers in monkey visual cortex. Exp Brain Res. 1983;50(1):133-145.

92. Bauer R, Dow DB, Vautin RG. Laminar distribution of preferred orientations in foveal striate cortex of the monkey. Exp Brain Res. 1980;41(1):54-60.

93. Bauer R, Dow BM. Complementary global maps for orientation coding in upper and lower layers of the monkey's foveal striate cortex. Exp Brain Res. 1989;76(3):503-509.

94. Murphy PC, Murphy, Sillito AM. Continuity of orientation columns between superficial and deep laminae of the cat primary visual cortex. J Physiol. 1986;381(1):95-110.
Eye and Brain

\section{Publish your work in this journal}

Eye and Brain is an international, peer-reviewed, open access journal focusing on clinical and experimental research in the field of neuro-ophthalmology. All aspects of patient care are addressed within the journal as well as basic research. Papers covering original research, basic science, clinical and epidemiological studies, reviews and

\section{Dovepress}

evaluations, guidelines, expert opinion and commentary, case reports and extended reports are welcome. The manuscript management system is completely online and includes a very quick and fair peer-review system, which is all easy to use. Visit http://www.dovepress.com/ testimonials.php to read real quotes from published authors. 